\title{
Software Product Line Oriented Feature Map
}

\author{
Yiyuan Li, Jianwei Yin, Dongcai Shi, Ying Li, and Jinxiang Dong \\ College of Computer Science and Technology, Zhejiang Univ., Hangzhou 310027, China \\ zjulyy@yahoo.com.cn, zjuyjw@zju.edu.cn, shidcai@163.com, \\ cnliying@zju.edu.cn, djx@zju.edu.cn
}

\begin{abstract}
The core idea of software product line engineering is to develop a reusable infrastructure that supports the software development of a family of products. On the base of domain analysis, feature modeling identifies commonalities and variability of software products in terms of features to provide an acknowledged abstract to various stakeholders. The concept of feature map is proposed to perfect feature model. It supports customized feature dependencies and constraint expresses, provides the capability to navigate and locate the resource entities of features. Ontology is introduced as the representation basis for the meta-model of feature maps. By the means of selecting features to construct the reusable infrastructure, the components of feature implementation are rapidly located and assembled to produce a family of software products meeting certain dependencies and constraints.
\end{abstract}

Keywords: Variability, Feature map, Resource navigation, Ontology.

\section{Introduction}

Currently the manufacture of software is suffering from such problems as individual customized requirements and frequent changes of business requirements. As a result, it seems that traditional software development mode - which is to develop software product specifically for certain application's requirements - costs more and has less efficiency and maintainability. In this software development mode, it's hard to meet the requirements of software development in large scale customization environment. The purpose of software production for mass customization is to produce and maintain a family of software products with similar functions, figure out both their commonalities and variability and manage these features [1]. It represents the trend of software factory's evolution.

Software product line is an effective way to implement software production for mass customization. It's a set of software systems with common controllable features. The core idea of software product line engineering is to develop a reusable infrastructure that supports the software development of a family of products [2]. A software product line typically consists of a product line architecture, a set of components and a set of products [3]. The characteristics of software development applying software product line principals are to maintain the common software assets and reuse them during the development process, such as domain model, software 
architecture, process model, components, etc. Each product derives its architecture from the product line architecture, instantiates and configures a subset of the product line components and usually contains some product-specific code. Instantiated products constitute a family of software products in domain.

Feature modeling is the mainstream of domain analysis for the software product line. Its main purpose is to identify all commonalities and variability in software product line. The outputs of feature modeling are all potential products of product line [4]. FORM [5] is a famous development method based on feature. Difference between domain products and family products shows the variability of software product line [2]. Variability point model [6,7] models the variability of software product line through four ways. The complex dependency relationships among variability points are presented in first order expression [8]. From the viewpoint of software configuration management, the variability management of software product line can be divided into nine sub modules according to two dimensions [9].

By analyzing the deficiency of current feature modeling and its description language, this paper proposes an expanded feature modeling of software product line - feature map. It perfects feature dependency description and restriction expression, supports quick navigation to feature resource artifacts of software product line in distributed collaborative development environment. Its meta-model is also presented.

\section{Feature Map}

Feature is the first-order entity in domain. It shows some capabilities or specialties owned by systems. It's the only determinate abstract in the domain and can be understood simultaneously by domain experts, users and developers. To a certain extent, feature is a kind of expression to ontology knowledge of application domain.

\subsection{Deficiency of Feature Model}

Feature modeling is to identify the commonalities and variability of all products in a software product line via analysis to domain features and their relationship. Domain reference architecture can be built according to feature model. The constituent units of the architecture can be bound with related component entities. However, existing feature model and its description technique have some deficiency.

Firstly, each domain may have its own feature mutual operation relation due to its variety. It has indetermination. Although existing feature models sum up and analyze the usual feature relation, they can not wholly describe all domain related feature dependency relation. Secondly, existing feature model trends to build feature model by aiming at domain systems' function. This forms the functional features. However, it seldom considers the non-functional domain features like performance, cost, and throughput etc. Also it lacks effective description and expression measure. Thirdly, domain feature analysis runs through all phases of software development lifecycle. It refers to a lot of resource entities like requirement specification, design model and component entities etc. Existing feature models only discuss production of software product from the viewpoint of feature selection. They ignore the problem of feature instantiation including the selection and locating of domain feature related resource entities. Fourthly, there may exist more than one component entity that implements 
the functions presented by a certain feature for choices. Existing feature models ignore the variability brought by feature implementation scheme.

Thus it can be seen that it is necessary to expand existing feature models to perfect the modeling and description ability for feature dependency relationship, nonfunctional feature constraint, feature resource navigation and variability of domain.

\subsection{Definition of Feature Map}

This paper proposes the concept of feature map. It supports feature dependency relationship and restriction expression and provides the capability of locating and navigating resource entities to implement feature selecting according to specified requirement, locate and assemble resource entities quickly and generate software product family that can satisfy dependency relationship and restriction conditions.

A feature map can be defined as a 5 elements set. $F M=\left(F, A, C, R, \lambda_{A}, \lambda_{C}, \lambda_{R}\right)$, among them,

- $F$ is the feature set of feature map;

- $A$ is the feature association set of feature map;

- $C$ is the feature constraint expression set of feature map;

- $R$ is the feature resource entity set of feature map;

- $\lambda_{A}$ denotes a mapping from $F$ to the set $P(A)$, i.e. $\lambda_{A}: F \rightarrow P(A) . P(A)$ represents the set of all the subsets of $A$. $\lambda_{A}$ meets the following conditions:

$$
\forall a \in A, \exists F^{\prime} \subset F,\left|F^{\prime}\right| \geq 2 \text { and } \forall f \in F^{\prime}, \lambda_{A}(f)=a
$$

This means that an arbitrary feature can have multiple dependency relationships with other features. Meanwhile, each feature association involves at least two features.

$-\lambda_{C}$ denotes a mapping from $F$ to the set $P(C)$, i.e. $\lambda_{C}: F \rightarrow P(C) . P(C)$ represents the set of all the subsets of $C$. $\lambda_{C}$ meets the following conditions:

$$
\forall c \in C, \exists F^{\prime} \subset F,\left|F^{\prime}\right| \geq 1 \text { and } \forall f \in F^{\prime}, \lambda_{c}(f)=c
$$

That is to say, for an arbitrary feature, it can be restricted by multiple constraint expressions; while each feature constraint can be specified to either a certain feature, or a set of features.

- $\lambda_{R}$ denotes a mapping from $F$ to the set $P(R)$, i.e. $\lambda_{R}: F \rightarrow P(R) . P(R)$ represents the set of all the subsets of $R$. $\lambda_{R}$ meets the following conditions:

$$
\forall f \neq f^{\prime} \in F, \lambda_{R}(f) \cap \lambda_{R}\left(f^{\prime}\right)=\varnothing \text { and } \bigcup \lambda_{R}(f)=R
$$

That is to say, each feature owns its resource entities.

Thus it can be concluded that the concept of feature map consists of two parts. On the one hand, feature map expands existing feature models to construct its infrastructure and foundation via perfecting feature dependency relationship definition of existing feature models and aggrandizing feature constraint expression to enhance the feature configuration relationship. On the other hand, feature map builds its superstructure via introducing the resource entities of features and providing the capability to rapidly navigate and locate them. With these two hands combined tightly, by the means of selecting features to construct the reusable infrastructure, the 
component entities of feature implementation are rapidly located and assembled to produce a family of software products meeting certain dependencies and constraints.

\subsection{Meta-model of Feature Map}

Features together with their dependency relationship, constrain expression and resource entities are abstracted as basic elements of meta-model. Corresponding with the web ontology language OWL, modeling elements of meta-model can be divided into ontology class element, object property element, data property element and data type element. Among them, ontology class element represents the semantic principal; object property element represents the association relationship among ontology class elements as the format of object property of ontology class elements, both its domain and range are ontology class elements; data property element represents the nonfunctional characteristics of ontology class element, its domain is ontology class element while its range is data type element.

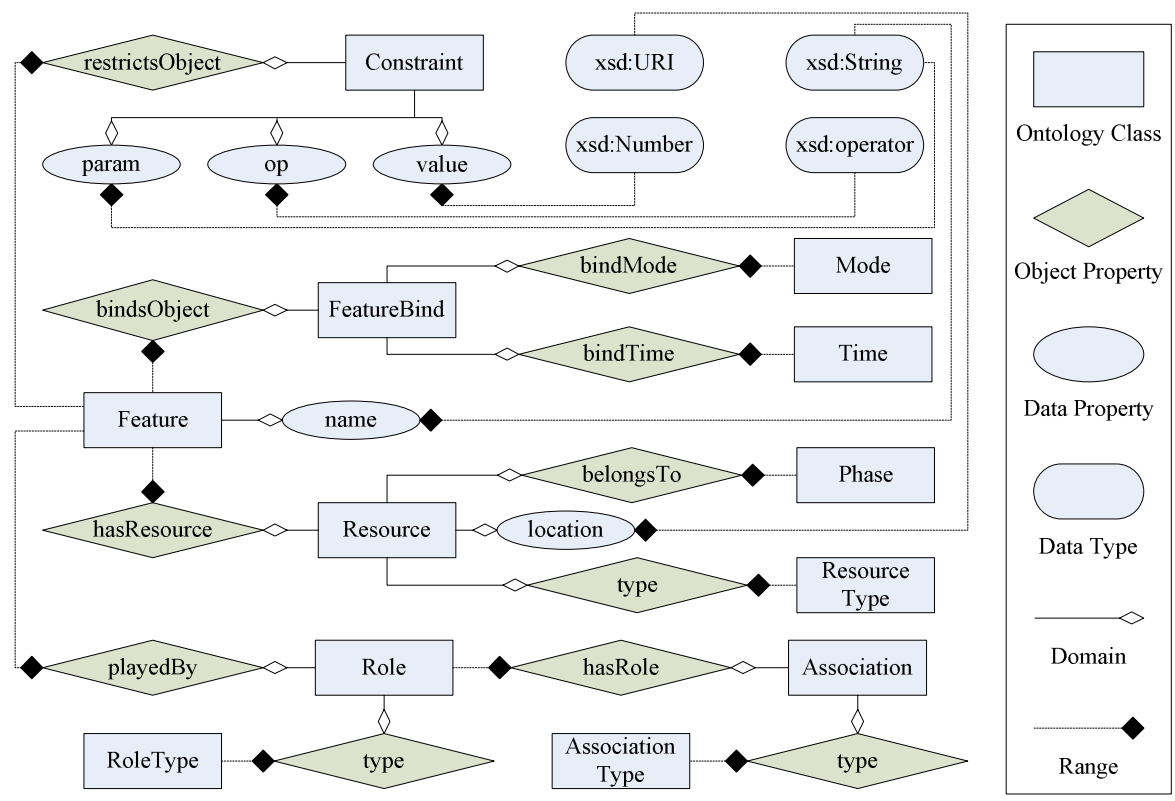

Fig. 1. The Meta Model of Feature Map Based on Ontology

The meta model of feature map based on ontology is described as figure 1, Feature, FeatureBind, Association, Constraint and Resource etc. are defined as ontology classes; while restrictsObject, hasResource, playedBy and hasRole etc. are defined as ontology object properties to establish the relation network of semantic principal; name, param, and location etc. are defined as data properties to describe the feature properties of semantic principal. The meanings of the main meta-model elements are described as following: 
Feature: ontology expression of feature definition in feature map, it's common or variable system characteristic that can be observed externally. Feature ontology instance is identified by a unique global name.

FeatureBind: ontology class of feature binding, it associates the binding mode and binding time through bindMode and bindTime object properties respectively.

Mode: binding mode of feature, including mandatory, optional, or, alternative and exclude etc. modes. Classified from the viewpoint of if this binding mode is affected by that of other features, mandatory and optional are unary binding modes while or, alternative and exclude etc. are multiple binding modes. However, if it's classified from the viewpoint of the variability of features, only features indicated by mandatory are the common indispensables while the ones indicated by others are optional features rested with the specific software products.

Time: binding time of feature, it only makes sense to the variable features that are indicated by optional, or, alternative and exclude etc. Its value can be design-time, compile-time, implement-time, assemble-time, load-time, instantiate-time, runtime etc.

Resource: expression of feature resource. It marks the software product development phase producing the resource via the association of belongsTo object property and Phase ontology class. It also indicates the type of entity object quoted by the resource via the association of type object property and ResourceType ontology class. Resource type is decided by the phase of software product development. Entities quoted by the resource may locate on any places in the distributed network environment, and can be navigated by URI through location object property.

Phase: stages of software product development. It includes requirement, design, implementation, test and maintenance etc. Although software product line engineering based on feature modeling is macroscopically similar with the traditional software engineering which is oriented to single software product development in the aspect of defining the phases of software development, they are dramatically different in the aspect of concrete actualizing approach and detail in each phase [10].

ResourceType: it can be requirements analysis document, model / flow design or component artifacts etc. This depends on the phase of software product development during which this resource is produced.

Constraint: the non-functional restrictions on feature. Constraint expression consists of a set of parameters, operators and variables. Constraint builds association with Feature ontology class through restrictObject object property and confirms the restricted object. Constraint can be defined to aim at property set of a single feature. It also can include multiple features as restricted objects and build feature constraint relationship under the general restriction.

Association: relationships between features. It associates the AssociationType ontology class through the type object property to confirm relation type. It also associates Role ontology class through the hasRole object property to make certain the objects referred by association. It is built based on at least two associated objects.

AssociationType: type of association, including composed-of, implemented-by, require, generalization/specialization and activate etc. Association has orientations, among which, composed-of, generalization/specialization and implemented-by belong to structural association; require and activate belong to reference association. 
Role: the object referred by association. It associates Feature ontology class through playedBy object property to make certain the real feature that assumes the role. It also associates RoleType ontology class via type object property to indicate the deserved role type. Assigning of role type determines the orientation of association.

RoleType: type of role. Its real range is decided by the type of association accompanying with the role.

The hierarchy of feature map is built by relationships like composed-of, generalization/specialization, implemented-by etc. among features. Common features are represented by setting binding mode to mandatory while variable features are established by marking the binding mode as optional, or, alternative or exclude etc. On the one hand, dependency and mutual operation among features are expressed by associations like implemented-by, require and activate etc. Moreover, the orientations of associations are determined by the role that feature takes within association. On the other hand, constraint expressions are built on the properties of a single feature or the properties set of feature group. All kinds of resource entities related to features in each development phase are navigated in network environment by location. Through this way, structural association, dependency association and constraint conditions among features are completely established. Meanwhile, by adding instances of AssociationType, RoleType and ResoureType, meta-model can describe the new associations and locate the new resource entities. Thus expansibility is available.

The variability of feature map is represented in several aspects. Firstly, as far as binding mode and binding time are concerned, the former directly determines whether the feature is selected or not, while the latter determines the occasion when the optional features are instantiated. Secondly, the relations among features like require and activate etc. determine if the other features that have dependency association or mutual operation association with the present feature will be selected or not. Thirdly, constraint expression determines the quantification constraint on the properties set of a single feature or feature group, and furthermore, it will affect the selection of component entities for feature implementation. Fourthly, on the base of the navigation and locating of resource entities, software products instantiated by selecting resource entities with same functions but different implementation plan will have different non-functional characteristics like performance and quality of service etc.

\section{Case Study}

Figure 2 shows the feature map of mobile telephone software product line and the mapping to its meta-model. Mobile telephone software product line is composed of some functional features like password protection, game, telephone directory and browser etc. Among them, password protection and browser are optional features. Meanwhile, multiple games can be the choice, but to some limitation, such as a small memory capacity, G3 and G4 can only be chosen one arbitrarily. In order to be in operation, the length of password should be set to 6 , the length of list in the telephone directory should be no more than 250 , and the required memory of embedded browser should be less than $2 \mathrm{M}$. In the process of feature analysis, each function feature has related requirements specification, design model and implementation component. Some functional features, for example, G2, even have various implementation schemes. 
Functional features like password protection, game, telephone book and browser etc are modeling as Feature ontology; the selection of feature is mandatory or optional is modeling as Mode ontology; max length of password, volume of telephone book and memory consumed by browser etc. are modeling as Constraint ontology; hierarchy structure of features and the mutually exclusive relationship between G3 and G4 etc. are modeling as Association ontology; requirements document, design models and component entities are modeling as ResourceType ontology; all lifecycle phases of software development are modeling as Phase ontology. The whole infrastructure of feature map is constructed by the associations among ontology via object properties, while the superstructure of feature map is constructed by modeling the reference of resource as location property to navigate and locate the resource entities.

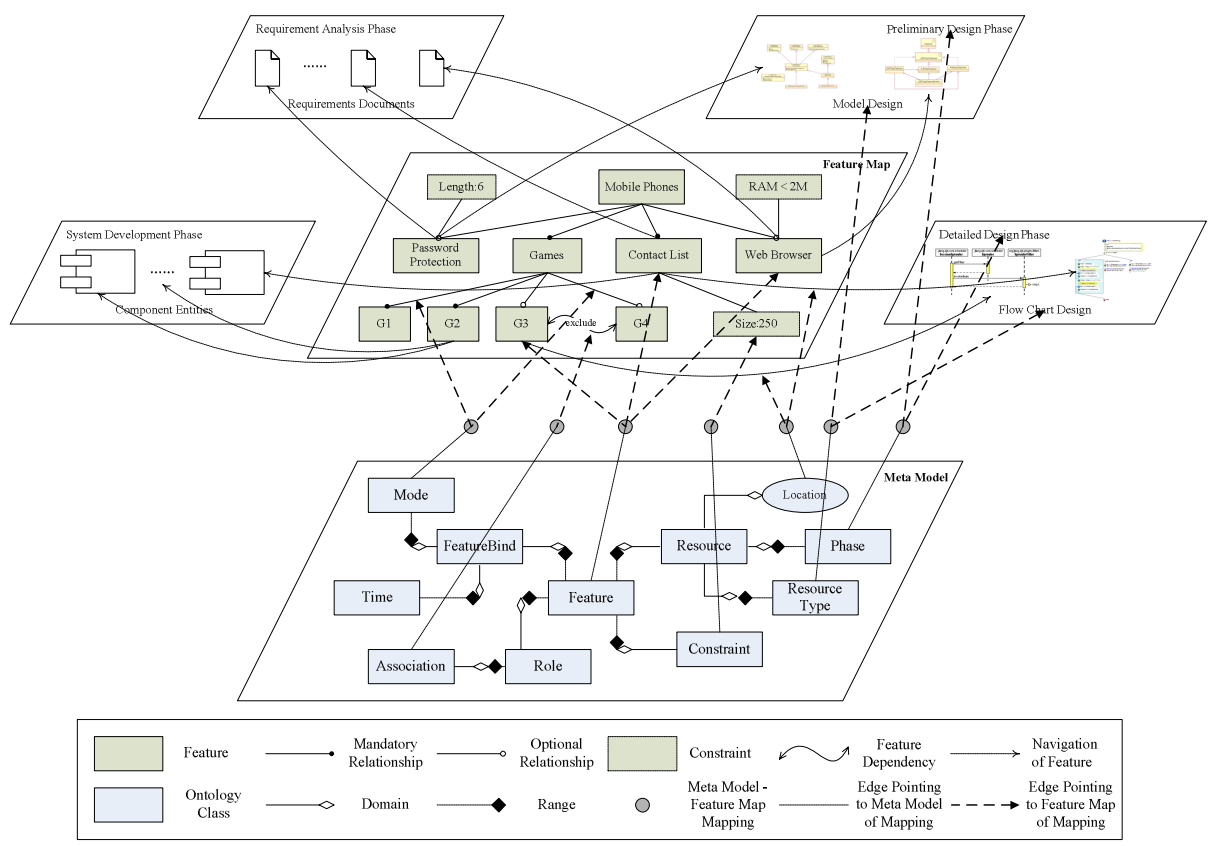

Fig. 2. Feature Map and Its Meta-model of Mobile Telephone Software Product Line

\section{Conclusion}

The core idea of software product line engineering is to develop a reusable infrastructure that supports the development of a family of software products. It's an efficient way to implement mass customized software production. Feature modeling is the mainstream of domain analysis of software product line. It identifies commonalities and variability of the products of a product line in terms of features to provide an acknowledged abstract to various stakeholders. Uncertainty of variable features determines the variability of software product line. Existing feature models and their description can not entirely support the diversity of feature dependencies in 
different domains. They do not support modeling and description of constraint expression and can not navigate and locate the resources in network environment. Moreover, their variability analysis did not consider the alternative of component entities which implement the features. In this paper, the concept of feature map is proposed to perfect feature model. Ontology is introduced as the representation basis for the meta-model of feature map. Feature map supports customized feature dependencies and constraint expressions, provides the capability to navigate and locate the resource entities of features. Then by the means of selecting features to construct the reusable infrastructure, the components of feature implementation are rapidly located and assembled to produce a family of software products meeting certain dependencies and constraints. The further work is to refine the feature map during studies and practices, including how to define and describe its related action characters and state transfer etc.

\section{References}

1. Charles W. Krueger. "Software Mass Customization". BigLever Software, Inc. (2001)

2. Michel Jaring, Jan Bosch. "Representing Variability in Software Product Lines: A Case Study". Proceedings of the 2th International Conference on Software Product Lines (SPLC'02), Springer Verlag LNCS 2379 (2002) 15-36

3. J. Bosch. "Design \& Use of Software Architectures - Adopting and Evolving a ProductLine Approach". Addison-Wesley (2000)

4. David Benavides, Pablo Trinidad, Antonio Ruiz-Cortes. "Automated Reasoning on Feature Models". Proceedings of the 17th International Conference on Advanced Information Systems Engineering (CAiSE'05), Springer Verlag LNCS 3520 (2005) 491-503

5. Kang KC, Kim S, Lee J, Kim K, Shin E, Huh M. "FORM: A Feature-Oriented Reuse Method with Domain-Specific Reference Architectures". Annals of Software Engineering (1998) 143-168

6. Jan Bosch, Gert Florijn, Danny Greefhorst. "Variability Issues in Software Product Lines". Proceedings of the 4th International Workshop on Software Product Family Engineering (PFE'02), Springer Verlag LNCS 2290 (2002) 13-21

7. Diana L. Webber, Hassan Gomaa. "Modeling Variability in Software Product Lines with The Variant Point Model". Elsevier (2003)

8. Macro Sinnema, Sybren Deelstra, Jos Nijhuis, Jan Bosch. "COVAMOF: A Framework for Modeling Variability in Software Product Families". Proceedings of the 3th International Conference on Software Product Lines (SPLC'04), Springer Verlag LNCS 3154 (2004) 197-213

9. Charles W. Krueger. "Variation Management for Software Production Lines". Proceedings of the 2th International Conference on Software Product Lines (SPLC'02), Springer Verlag LNCS 2379 (2002) 37-48

10. Kyo C. Kang, Jaejoon Lee, Patrick Donohoe. "Feature-Oriented Product Line Engineering”. IEEE Software, Volume 19, Issue 4, July-Aug (2002) 58-65 